\title{
Carta al Director: la enfermería de PRÁCTICA AVANZADA: ¿ES LA ESTRATEGIA PARA ALCANZAR EL ACCESO Y COBERTURA UNIVERSAL EN SALUD?
}

\section{ADVANCED PRACTICE NURSE: IS THE STRATEGY TO ACHIEVE UNIVERSAL HEALTH ACCESS AND COVERAGE?}

Tras leer las importantes apreciaciones realizadas por Sevilla Guerra (1) en el artículo de revisión "Enfermeras como sustitutas de médicos en atención primaria" publicado en el Vol.13 $\mathrm{N}^{\circ} 1$ (2019) me permito realizar esta complementación basándome en la evidencia disponible y al final poniendo en breve contexto la situación de Sudamérica.

La enfermería a lo largo de la historia ha evolucionado de una forma considerable, desde el paso de ser un arte, hasta hoy postularse como una ciencia; esta importante transición que ha supuesto la enfermería hace hincapié a la vasta capacidad que tienen las/os enfermeras/os de adquirir conocimiento y habilidades para brindar cuidados satisfactorios que están a la vanguardia con el evolucionar de la salud.

En la actualidad, la mayor preocupación de las organizaciones que rigen la salud a nivel de las Américas es el aumento desmesurado de las tasas de natalidad y envejecimiento, enfermedades crónico degenerativas, enfermedades infectocontagiosas, etc., esto sumado a que la escasez de personal sanitario en las Américas estimado por la Organización Mundial de la Salud es de casi 800.000 profesionales (2) genera un problema, sobre todo cuando se tiene como objetivo primordial "brindar el acceso y cobertura universal en salud a todas las poblaciones".

En el año 2013, los estados miembros de la Organización Panamericana de Salud (OPS), la Oficina Regional de la Organización Mundial de la Salud para las Américas, aprobó la Resolución CD52.R13 de Recursos Humanos para la Salud: Aumentar el acceso al personal sanitario capacitado en sistemas de salud basados en la atención primaria $(3,4)$; en la misma línea en el 2014, se aprueba la Resolución CD53/52 para el 
Acceso Universal a la Salud y Cobertura Universal de Salud dejando en constancia 4 ejes: a) aumentar el acceso al personal sanitario capacitado para la APS, b) brindar apoyo para mejorar el alcance de la práctica de cada profesión hasta su máximo potencial incluyendo los especialistas clínicos que no son médicos, c) que introduzcan nuevos perfiles profesionales y técnicos y fortalezcan los existentes, d) enfermeras expertas en la APS podrían apoyar eficazmente para lograr alcanzar el acceso universal en salud (5).

En referencia al último ítem, cabe señalar que la enfermería de práctica avanzada (EPA) ya ha existido desde la década de 1970 en los Estados Unidos y Canadá, países en los cuales aun se mantiene esta figura. La EPA es definida por el Consejo Internacional de Enfermería (CIE) como "...una enfermera que ha adquirido la base del conocimiento experto, capaz de tomar decisiones complejas durante su quehacer, con competencias clínicas para una práctica expandida, cuyas características están determinadas por el contexto y/o el país en el que esta enfermera(o) está acreditada(o) para ejercer. Se recomienda un nivel de magister para la formación de estos profesionales". Es decir son profesionales con un alto nivel de autonomía profesional e independencia en la práctica, ges- tión de casos, habilidades avanzadas en valoración de salud, habilidades en toma de decisiones y capacidad de razonamiento diagnóstico. Reconocidas competencias clínicas avanzadas, capaces de brindar consultoría a otros proveedores de salud, capaz de planificar, implementar y evaluar programas y ser reconocida como primer punto de contacto para los pacientes (6).

A pesar de haber existido la EPA desde hace varias décadas, en los últimos años se ha visto la necesidad de ampliar el rol de las enfermeras y enfermeros en la región, donde constituyen la gran mayoría del personal de salud, como lo señala James Fitzgerald, director del Departamento de Sistemas y Servicios de Salud de la OPS (2). Como mencioné anteriormente, EE.UU y Canadá son los países de América que cuentan con la EPA establecida, con otros países como México que ha entrado en ese camino al permitir a las enfermeras (os) prescribir medicamentos ante la ausencia del médico, y en situaciones de urgencia, así mismo, Argentina, Brasil, Chile, Colombia, México, Panamá y Perú quienes poseen un alto grado de acceso a la educación de posgrado en enfermería y que podrían en el futuro ofrecer la formación necesaria a las EPA.

En la actualidad el debate esta abierto y las sugerencias por parte de la 
OPS/OMS están dadas, tanto así que en el año 2015 la Universidad de McMaster y la OPS organizaron una Cumbre de Enfermería de Práctica Avanzada (6) con el objetivo de promover la ampliación del alcance de la práctica de enfermería en atención primaria de salud para América Latina y el Caribe, en donde se insta a todos los ministerios de salud de los diferentes países a buscar y aplicar medidas en función de poner en marcha esta estrategia.

Una revisión del rol de la EPA aplicada a la APS, realizada en Australia, Bélgica, Canadá, Chipre, Estados Unidos de América, Finlandia, Francia, Irlanda, Japón, Polonia, el Reino Unido y la República Checa, mostró que las enfermeras pueden contribuir a mejorar el acceso a los servicios de salud y a reducir los tiempos de espera de los usuarios (7). Asimismo, se encontró que las EPA pueden ofrecer la misma calidad de atención que los médicos y que ésta es una práctica eficiente para los programas que requieren seguimiento de rutina $(7,8)$.

La existencia de la EPA supondría un importante avance en la atención, acceso y cobertura de salud que se da a la población, ya que, como se mencionó anteriormente el contingente enfermero es la mayor fuerza laboral del sistema de salud por lo que no es descabellado pensar en que si en las diferentes unidades asistenciales de salud (puerta de entrada al sistema) se cuenta con EPA capaces de brindar una atención de salud óptima en conjunto con el equipo multidisciplinario se podría abarcar una mayor cantidad de población atendida y por ende mejorar el acceso a la salud, y como la estrategia se inicia en el primer nivel, la atención en los demás niveles mejoraría de forma exponencial, ya que, como lo menciona la OMS (9) "las funciones o los roles más avanzados de las enfermeras se han implementado para: mejorar el acceso a la atención en zonas con suministro limitado de médicos; maximizar el acceso a la atención primaria en salud, y permitir un seguimiento intensivo de pacientes con enfermedades crónicas en atención primaria".

Como comentario final es importante mencionar que la implementación de la EPA en Sudamérica debe ir de la mano con: el mejoramiento de los planes de estudio que ofertan las diferentes universidades en la formación de pregrado y el aumento de post grados en enfermería; con el cambio del modelo del Sistema de Salud actual de los países por un sistema flexible, enfocado en la prevención de enfermedades como su pilar fundamental; con el empoderamiento de las/ los profesionales desde las aulas universitarias, visionando a la enfermería como 
un campo de acción amplio y autónomo y no simplemente subordinado a la labor médica. Finalmente respondo a la interrogante planteada en el título: La enfermería de práctica avanzada: ¿es la estrategia para alcanzar el acceso y cobertura universal en salud?: Si.

Lic. Jonathan Velasco Yánez 


\section{BiBLIOGRAFÍA}

1. Sevilla-Guerra S. ENFERMERAS COMO SUSTITUTAS DE MÉDICOS EN ATENCIÓN PRIMARIA (REVISIÓN). Revista ENE de Enfermería [Internet]. 27 de marzo de 2019 [citado 21 de mayo de 2019];13(1). Disponible en: http://ene-enfermeria.org/ojs/index.php/ENE/article/view/963

2. OPS/OMS Ecuador. OPS/OMS Ecuador OPS insta a ampliar el rol de las enfermeras en la atención primaria de salud I OPS/OMS [Internet]. Pan American Health Organization / World Health Organization. 2018 [citado 20 de mayo de 2019]. Disponible en: https://www.paho.org/ecu/index.php? option=com_content $\&$ view $=$ article $\& i d=2024$ :ops-insta-a-ampliar-el-rol-de-las-enfermeras-en-la-atencionprimaria-de-salud\&ltemid $=360$

3. Pan American Health Organization. Resolution CD52.R13 Human Resources for Health: Increasing Access to Qualified Health Workers In Primary Health Care-Based Health System. [Internet]. 2013. Disponible en: https://www.paho.org/hq/dmdocuments/2013/CD52-R13-e.pdf

4. Cassiani SHDB, Rosales LK, Cassiani SHDB, Rosales LK. Iniciativas para la Implementación de la Enfermería de Práctica Avanzada en la Región de las Américas. Escola Anna Nery [Internet]. 2016 [citado 20 de mayo de 2019];20(4). Disponible en: http://www.scielo.br/scielo.php?script=sci_abstract\&pid=S1414-81452016000400101\&Ing=en\&nrm =iso\&tlng=es

5. Asociación Chilena de educación en enfermería. La enfermería de práctica avanzada como una estrategia para alcanzar el acceso y cobertura universal de salud. En Santiago, Chile; 2016. Disponible en: https://www.paho.org/hq/index.php? option=com_docman\&view=download\&category_sI u g = p u b I i c a c i ones - t e c n i c a s cientificas-7983\&alias $=40061$-reporte-simposio-enfermeria-practica-avanzada-como-una-estrategiaalcanzar-acceso-cobertura-universal-salud-santiagoc h i l e - $2016-v$ e r s i o n espanol-061\&ltemid=270\&lang=es

6. School of nursing University of Michigan, World Health Organization. Desarrollando competencias de enfermería de práctica avanzada en Latinoamérica para contribuir a la salud universal. En Michigan, EE.UU; 2016. Disponible en: https://www.$\mathrm{p} a \mathrm{~h}$ o. or g/h q/i $\mathrm{ndex}$. ph p ? option=com_docman\&view=download\&category_sI $u g=p u b \mid$ i c a c i o n e s - t e c n i c a s cientificas-7983\&alias $=40048$-informe-cumbre-enfermeria-practica-avanzada-desarrollando-competencias-enfermeria-practica-avanzada-latinoamericacontribuir-a-salud-universal-ann-arbor-usa-2016-version-espanol-048\&ltemid=270\&lang=es

7. Delamaire M-L, Lafortune G. Nurses in Advanced Roles. OECD Health Working Papers
[Internet]. 8 de julio de 2010 [citado 20 de mayo de 2019]; Disponible en: https://www.oecd-ilibrary.org/ social-issues-migration-health/nurses-in-advancedroles_5kmbrcfms5g7-en

8. Martínez-González NA, Tandjung R, Djalali $\mathrm{S}$, Rosemann $\mathrm{T}$. The impact of physician-nurse task shifting in primary care on the course of disease: a systematic review. Human Resources for Health. 7 de julio de 2015;13(1):55.

9. World Health Organization. WHO nursing and midwifery progress report, 2008-2012. [Internet]. World Health Organization; 2013 [citado 25 de junio de 2019]. Disponible en: http://www.who.int/hrh/nursing_midwifery/NursingMidwiferyProgressReport.pdf 